\title{
SOME PROPERTIES OF A SEQUENCE ANALOGOUS TO EULER NUMBERS
}

\section{ZHI-HONG SUN}

(Received 28 April 2012; accepted 6 May 2012; first published online 12 June 2012)

\section{Abstract}

Let $\left\{U_{n}\right\}$ be given by $U_{0}=1$ and $U_{n}=-2 \sum_{k=1}^{[n / 2]}\left(\begin{array}{c}n \\ 2 k\end{array}\right) U_{n-2 k}(n \geq 1)$, where [-] is the greatest integer function. Then $\left\{U_{n}\right\}$ is analogous to the Euler numbers and $U_{2 n}=3^{2 n} E_{2 n}\left(\frac{1}{3}\right)$, where $E_{m}(x)$ is the Euler polynomial. In a previous paper we gave many properties of $\left\{U_{n}\right\}$. In this paper we present a summation formula and several congruences involving $\left\{U_{n}\right\}$.

2010 Mathematics subject classification: primary 11A07; secondary 11B68.

Keywords and phrases: congruence, summation formula, Euler number.

\section{Introduction}

The Euler numbers $\left\{E_{n}\right\}$ and Euler polynomials $\left\{E_{n}(x)\right\}$ are defined by

$$
\begin{gathered}
E_{0}=1, \quad E_{n}=-\sum_{k=1}^{[n / 2]}\left(\begin{array}{c}
n \\
2 k
\end{array}\right) E_{n-2 k} \quad(n \geq 1), \\
E_{n}(x)=\frac{1}{2^{n}} \sum_{k=0}^{[n / 2]}\left(\begin{array}{c}
n \\
2 k
\end{array}\right)(2 x-1)^{n-2 k} E_{2 k} \quad(n \geq 0),
\end{gathered}
$$

where $[x]$ is the greatest integer not exceeding $x$. In [8] the author introduced and studied the sequence $\left\{U_{n}\right\}$ (similar to Euler numbers) given by

$$
U_{0}=1, \quad U_{n}=-2 \sum_{k=1}^{[n / 2]}\left(\begin{array}{c}
n \\
2 k
\end{array}\right) U_{n-2 k} \quad(n \geq 1) .
$$

Since $U_{1}=0$, by induction $U_{2 n-1}=0$ for $n \geq 1$. The first few values of $U_{2 n}$ are as follows:

$$
\begin{gathered}
U_{2}=-2, \quad U_{4}=22, \quad U_{6}=-602, \quad U_{8}=30742, \quad U_{10}=-2523002, \\
U_{12}=303692662, \quad U_{14}=-50402079002, \quad U_{16}=11030684333782 .
\end{gathered}
$$

The author is supported by the Natural Sciences Foundation of China (grant no. 10971078).

(C) 2012 Australian Mathematical Publishing Association Inc. 0004-9727/2012 \$16.00 
Let $(a / p)$ be the Legendre symbol. In [8], the author proved that for any prime $p>3$,

$$
\sum_{k=1}^{[2 p / 3]} \frac{(-1)^{k-1}}{k} \equiv 3 p\left(\frac{p}{3}\right) U_{p-3} \quad\left(\bmod p^{2}\right)
$$

The Bernoulli numbers $\left\{B_{n}\right\}$ and Bernoulli polynomials $\left\{B_{n}(x)\right\}$ are given by

$$
B_{0}=1, \quad \sum_{k=0}^{n-1}\left(\begin{array}{l}
n \\
k
\end{array}\right) B_{k}=0(n \geq 2) \quad \text { and } \quad B_{n}(x)=\sum_{k=0}^{n}\left(\begin{array}{l}
n \\
k
\end{array}\right) B_{k} x^{n-k}(n \geq 0) .
$$

By [8, p. 217],

$$
B_{p-2}\left(\frac{1}{3}\right) \equiv 6 U_{p-3} \quad(\bmod p) \quad \text { for any prime } p>3 .
$$

In [4] Mattarei and Tauraso proved that for any prime $p>3$,

$$
\sum_{k=0}^{p-1}\left(\begin{array}{c}
2 k \\
k
\end{array}\right) \equiv\left(\frac{p}{3}\right)-\frac{p^{2}}{3} B_{p-2}\left(\frac{1}{3}\right) \quad\left(\bmod p^{3}\right) .
$$

Thus,

$$
\sum_{k=0}^{p-1}\left(\begin{array}{c}
2 k \\
k
\end{array}\right) \equiv\left(\frac{p}{3}\right)-2 p^{2} U_{p-3} \quad\left(\bmod p^{3}\right) \quad \text { for any prime } p>3
$$

Suppose that $p$ is a prime of the form $3 k+1$ and so $4 p=L^{2}+27 M^{2}$ for some integers $L$ and $M$. Assume that $L \equiv 1(\bmod 3)$. From $(1.3)$ and [3, Theorem 6]

$$
\left(\begin{array}{c}
\frac{2(p-1)}{3} \\
\frac{p-1}{3}
\end{array}\right) \equiv\left(-L+\frac{p}{L}+\frac{p^{2}}{L^{3}}\right)\left(1+p^{2} U_{p-3}\right) \equiv-L+\frac{p}{L}+p^{2}\left(\frac{1}{L^{3}}-L U_{p-3}\right) \quad\left(\bmod p^{3}\right) .
$$

In Section 2 we prove a summation formula involving $U_{n}$; see Theorem 2.1. Let $\mathbb{N}$ be the set of positive integers. If $n \in \mathbb{N}$ and $2^{\alpha} \mid n$, in [8] the author determined $U_{2 n}$ $\left(\bmod 2^{\alpha+7}\right)$. In Section 3 we prove that

$$
3 U_{2 n} \equiv-3072 n^{4}+4608 n^{3}+2240 n^{2}+1680 n+2 \quad\left(\bmod 2^{\alpha+14}\right) \quad \text { for } n \geq 7 .
$$

For $k, m, b \in \mathbb{N}$ with $2 \mid b$, in Section 3 we also show that

$$
U_{2^{m} k+b} \equiv U_{b}+2^{b+1} \quad\left(\bmod 2^{\min \{b, m\}+3}\right) .
$$

Let $k, m \in \mathbb{N}$ and $b \in\{0,2,4, \ldots\}$. From [8, Theorem 4.3] we have $U_{k \varphi\left(3^{m}\right)+b} \equiv U_{b}$ $\left(\bmod 3^{m}\right)$, where $\varphi(n)$ is Euler's totient function. In Section 4 we prove a congruence for $U_{k \varphi\left(3^{m}\right)+b}-U_{b}\left(\bmod 3^{m+4}\right)$ for $m \geq 3$; see Theorem 4.2. In Section 5 we prove a congruence for $E_{k \varphi\left(3^{m}\right)+b}-\left(3^{b}+1\right) E_{b}\left(\bmod 3^{m+4}\right)$ for $m \geq 3$; see Theorem 5.2. 


\section{A summation formula involving $U_{n}(x)$}

For $n=0,1,2, \ldots$ let

$$
U_{n}(x)=\sum_{r=0}^{n}\left(\begin{array}{l}
n \\
r
\end{array}\right) U_{r} x^{n-r}=\sum_{k=0}^{[n / 2]}\left(\begin{array}{c}
n \\
2 k
\end{array}\right) U_{2 k} x^{n-2 k} .
$$

The first few $U_{n}(x)$ are as follows:

$$
\begin{gathered}
U_{0}(x)=1, \quad U_{1}(x)=x, \quad U_{2}(x)=x^{2}-2, \\
U_{3}(x)=x^{3}-6 x, \quad U_{4}(x)=x^{4}-12 x^{2}+22, \\
U_{5}(x)=x^{5}-20 x^{3}+110 x, \quad U_{6}(x)=x^{6}-30 x^{4}+330 x^{2}-602 .
\end{gathered}
$$

By [8, Theorem 2.3],

$$
\begin{gathered}
U_{n}(x-1)-U_{n}(x)+U_{n}(x+1)=x^{n}, \\
U_{n}(x)+U_{n}(x+3)=(x+1)^{n}+(x+2)^{n}, \\
U_{n}(x+3)-U_{n}(x-3)=(x+2)^{n}+(x+1)^{n}-(x-1)^{n}-(x-2)^{n} .
\end{gathered}
$$

Taking $a_{n}=U_{n}(x)$ and $b_{n}=x^{n}$ in [8, Theorem 2.2],

$$
x^{n}=2 \sum_{k=0}^{[n / 2]}\left(\begin{array}{c}
n \\
2 k
\end{array}\right) U_{n-2 k}(x)-U_{n}(x) .
$$

That is,

$$
U_{n}(x)=x^{n}-2 \sum_{k=1}^{[n / 2]}\left(\begin{array}{c}
n \\
2 k
\end{array}\right) U_{n-2 k}(x)
$$

Since

$$
\begin{aligned}
\int_{a}^{b} U_{n}(x) d x & =\sum_{k=0}^{n}\left(\begin{array}{l}
n \\
k
\end{array}\right) U_{k} \int_{a}^{b} x^{n-k} d x=\left.\sum_{k=0}^{n}\left(\begin{array}{l}
n \\
k
\end{array}\right) U_{k} \frac{x^{n-k+1}}{n-k+1}\right|_{a} ^{b} \\
& =\left.\frac{1}{n+1} \sum_{k=0}^{n+1}\left(\begin{array}{c}
n+1 \\
k
\end{array}\right) U_{k} x^{n+1-k}\right|_{a} ^{b}
\end{aligned}
$$

we see that

$$
\int_{a}^{b} U_{n}(x) d x=\frac{U_{n+1}(b)-U_{n+1}(a)}{n+1} .
$$

This together with (2.3) yields

$$
\int_{a-3}^{a+3} U_{n}(x) d x=\frac{(a+2)^{n+1}+(a+1)^{n+1}-(a-1)^{n+1}-(a-2)^{n+1}}{n+1} .
$$


Since $U_{n}(0)=U_{n}$, by (2.4),

$$
U_{n}(x)=U_{n}+n \int_{0}^{x} U_{n-1}(t) d t .
$$

Let $m, n \in \mathbb{N}$. From [1] we have the following well-known summation formulas:

$$
\sum_{k=0}^{m-1} k^{n}=\frac{B_{n+1}(m)-B_{n+1}}{n+1} \text { and } \sum_{k=0}^{m-1}(-1)^{k} k^{n}=\frac{E_{n}(0)-(-1)^{m} E_{n}(m)}{2} .
$$

We now present the following similar result.

Theorem 2.1. Let $m, n \in \mathbb{N}$ and

$$
S_{n}(m)=(m-1)^{n}+(m-2)^{n}-(m-4)^{n}-(m-5)^{n}+(m-7)^{n}+(m-8)^{n}-\cdots,
$$

where the term $a^{n}$ vanishes when $a \leq 0$. Then

$$
S_{n}(m)= \begin{cases}U_{n}(m)-(-1)^{m / 3} U_{n} & \text { if } 3 \mid m, \\ U_{n}(m)-(-1)^{[(m+1) / 3]} U_{n} / 2 & \text { if } 3 \nmid m \text { and } 2 \mid n, \\ U_{n}(m)-(-1)^{[m / 3]} U_{n}(1) & \text { if } 3 \nmid m \text { and } 2 \nmid n .\end{cases}
$$

Proof. Using (2.2), we see that

$$
\begin{aligned}
(m-1)^{n}+(m-2)^{n}-(m-4)^{n}-(m-5)^{n}+(m-7)^{n}+(m-8)^{n} \\
-\cdots-(-1)^{[m / 3]}\left(\left(m-3\left[\frac{m}{3}\right]+2\right)^{n}+\left(m-3\left[\frac{m}{3}\right]+1\right)^{n}\right) \\
=\left(U_{n}(m)+U_{n}(m-3)\right)-\left(U_{n}(m-3)+U_{n}(m-6)\right)+\left(U_{n}(m-6)+U_{n}(m-9)\right) \\
\quad-\cdots-(-1)^{[m / 3]}\left(U_{n}\left(m-3\left[\frac{m}{3}\right]+3\right)+U_{n}\left(m-3\left[\frac{m}{3}\right]\right)\right) \\
=U_{n}(m)-(-1)^{[m / 3]} U_{n}\left(m-3\left[\frac{m}{3}\right]\right) .
\end{aligned}
$$

Thus,

$$
S_{n}(m)= \begin{cases}U_{n}(m)-(-1)^{m / 3} U_{n}(0) & \text { if } 3 \mid m, \\ U_{n}(m)-(-1)^{[m / 3]} U_{n}(1) & \text { if } 3 \mid m-1, \\ (-1)^{[m / 3]} \cdot 1+U_{n}(m)-(-1)^{[m / 3]} U_{n}(2) & \text { if } 3 \mid m-2 .\end{cases}
$$

Clearly $U_{n}(0)=U_{n}$. By (2.2) and (2.1), we have $U_{n}(-1)+U_{n}(2)=1$ and so $U_{n}(2)=$ $1-U_{n}(-1)=1-(-1)^{n} U_{n}(1)$. If $2 \mid n$, using (1.1) we see that

$$
U_{n}(1)=\sum_{k=0}^{n / 2}\left(\begin{array}{c}
n \\
2 k
\end{array}\right) U_{2 k}=\sum_{k=0}^{n / 2}\left(\begin{array}{c}
n \\
2 k
\end{array}\right) U_{n-2 k}=U_{n}-\frac{1}{2} U_{n}=\frac{1}{2} U_{n}
$$

and so

$$
U_{n}(2)=1-U_{n}(1)=1-\frac{1}{2} U_{n} .
$$

Now putting all the above together, we deduce the result. 
Corollary 2.2. For $m \in \mathbb{N}$,

$$
\begin{gathered}
S_{2}(m)= \begin{cases}m^{2}-2+2(-1)^{m / 3} & \text { if } 3 \mid m, \\
\left.m^{2}-2+(-1)^{[(m+1) / 3}\right] & \text { if } 3 \nmid m,\end{cases} \\
S_{3}(m)= \begin{cases}m^{3}-6 m & \text { if } 3 \mid m, \\
m^{3}-6 m+5(-1)^{[m / 3]} & \text { if } 3 \nmid m,\end{cases} \\
S_{4}(m)= \begin{cases}m^{4}-12 m^{2}+22\left(1-(-1)^{m / 3}\right) & \text { if } 3 \mid m, \\
m^{4}-12 m^{2}+11\left(2-(-1)^{[(m+1) / 3]}\right) & \text { if } 3 \nmid m .\end{cases}
\end{gathered}
$$

Corollary 2.3. For $n \in \mathbb{N}$,

$$
\begin{aligned}
U_{2 n} & =\frac{2}{3}\left(2^{2 n}+3^{2 n}-\sum_{k=1}^{n}\left(\begin{array}{l}
2 n \\
2 k
\end{array}\right) 4^{2 k} U_{2 n-2 k}\right) \\
& =\frac{2}{3}\left(7^{2 n}+6^{2 n}-4^{2 n}-3^{2 n}+1-\sum_{k=1}^{n}\left(\begin{array}{l}
2 n \\
2 k
\end{array}\right) 8^{2 k} U_{2 n-2 k}\right) .
\end{aligned}
$$

Proof. Taking $m=4,8$ in Theorem 2.1 and replacing $n$ with $2 n$, we see that

$$
3^{2 n}+2^{2 n}=U_{2 n}(4)+\frac{1}{2} U_{2 n}
$$

and

$$
7^{2 n}+6^{2 n}-4^{2 n}-3^{2 n}+1=U_{2 n}(8)+\frac{1}{2} U_{2 n}
$$

Since

$$
U_{2 n}(x)=\sum_{r=0}^{n}\left(\begin{array}{l}
2 n \\
2 r
\end{array}\right) U_{2 r} x^{2 n-2 r}=U_{2 n}+\sum_{k=1}^{n}\left(\begin{array}{l}
2 n \\
2 k
\end{array}\right) x^{2 k} U_{2 n-2 k},
$$

from the above we deduce the result.

\section{Congruences for $U_{2 n}\left(\bmod 2^{14}\right)$ and $U_{2^{m}} k+b\left(\bmod 2^{\min \{b, m\}+3}\right)$}

Suppose that $n \in\{3,4,5, \ldots\}$. From [8, Theorem 4.1 and Corollary 4.1] we know that

$$
U_{2 n} \equiv-16 n-42 \quad\left(\bmod 2^{7}\right) .
$$

Moreover, if $n$ is even and $2^{\alpha} \mid n$, then

$$
U_{2 n} \equiv 48 n+\frac{2}{3} \quad\left(\bmod 2^{\alpha+7}\right) .
$$

Let $p$ be a prime and let $\operatorname{ord}_{p} m$ be the greatest integer $\alpha$ such that $p^{\alpha} \mid m$. If $p^{s} \leq n<p^{s+1}$, then

$$
\begin{aligned}
\operatorname{ord}_{p} n ! & =\left[\frac{n}{p}\right]+\left[\frac{n}{p^{2}}\right]+\cdots+\left[\frac{n}{p^{s}}\right]<\frac{n}{p}+\frac{n}{p^{2}}+\cdots+\frac{n}{p^{s}}+\cdots \\
& =n \cdot \frac{1 / p}{1-1 / p}=\frac{n}{p-1}
\end{aligned}
$$


Lemma 3.1. Suppose that $n \in \mathbb{N}, n \geq 5$ and $2^{\alpha} \mid n$. Then

$$
\begin{aligned}
3 U_{2 n}+2^{7} n(2 n-1) U_{2 n-2} \equiv & 2\left(7^{2 n}+6^{2 n}-4^{2 n}-3^{2 n}+1\right)+2^{16} n^{2}(n-1) \\
& -23 \cdot 2^{13} n(n-1)+7 \cdot 2^{15} n(n-1)^{3} \quad\left(\bmod 2^{\alpha+19}\right) .
\end{aligned}
$$

Proof. For $k \geq 4$ we see that $6(k-3)>k \geq \operatorname{ord}_{2} k$ and so $8^{2 k} / k=2^{18} \cdot 2^{6(k-3)} / k \equiv 0$ $\left(\bmod 2^{19}\right)$. For $3 \leq k \leq n-1$, by (1.1) we have $2 \mid U_{2 n-2 k}$. Thus, for $k \geq 3$,

$$
2\left(\begin{array}{c}
2 n \\
2 k
\end{array}\right) 8^{2 k} U_{2 n-2 k}=2 n\left(\begin{array}{c}
2 n-1 \\
2 k-1
\end{array}\right) \frac{8^{2 k}}{k} U_{2 n-2 k} \equiv 0 \quad\left(\bmod 2^{\alpha+20}\right) .
$$

Hence, by Corollary 2.3,

$$
3 U_{2 n} \equiv 2\left(7^{2 n}+6^{2 n}-4^{2 n}-3^{2 n}+1-\left(\begin{array}{c}
2 n \\
2
\end{array}\right) 8^{2} U_{2 n-2}-\left(\begin{array}{c}
2 n \\
4
\end{array}\right) 8^{4} U_{2 n-4}\right) \quad\left(\bmod 2^{\alpha+20}\right) .
$$

Since $U_{2 n-4} \equiv-16(n-2)-42=-16 n-10\left(\bmod 2^{7}\right)$ and

$$
2\left(\begin{array}{c}
2 n \\
4
\end{array}\right) 8^{4}=2^{12} n(n-1) \frac{4(n-1)^{2}-1}{3} \equiv 0 \quad\left(\bmod 2^{\alpha+12}\right),
$$

we see that

$$
\begin{aligned}
2\left(\begin{array}{c}
2 n \\
4
\end{array}\right) 8^{4} U_{2 n-4} & \equiv 2^{12} n(n-1) \frac{4(n-1)^{2}-1}{3}(-16 n-10) \\
& =-2^{16} n^{2}(n-1) \frac{4(n-1)^{2}-1}{3}-2^{13} n(n-1) \frac{5\left(4(n-1)^{2}-1\right)}{3} \\
& \equiv-2^{16} n^{2}(n-1) \cdot 3(4(n-1)-1)-23 \cdot 2^{13} n(n-1)\left(4(n-1)^{2}-1\right) \\
& \equiv-2^{16} n^{2} \cdot 3(4(n-1)-(n-1))+23 \cdot 2^{13} n(n-1)-23 \cdot 2^{15} n(n-1)^{3} \\
& \equiv-2^{16} n^{2}(n-1)+23 \cdot 2^{13} n(n-1)-7 \cdot 2^{15} n(n-1)^{3}\left(\bmod 2^{\alpha+19}\right) .
\end{aligned}
$$

Hence, by (3.3) and the fact $2\left(\begin{array}{c}2 n \\ 2\end{array}\right) 8^{2} U_{2 n-2}=2^{7} n(2 n-1) U_{2 n-2}$ we deduce the result.

Theorem 3.2. Let $n \in \mathbb{N}$ with $n \geq 7$ and $2^{\alpha} \mid n$. Then

$$
3 U_{2 n} \equiv-3072 n^{4}+4608 n^{3}+2240 n^{2}+1680 n+2\left(\bmod 2^{\alpha+14}\right) .
$$

Proof. Since $U_{2 n-2} \equiv-16(n-1)-42\left(\bmod 2^{7}\right)$, by Lemma 3.1,

$$
\begin{aligned}
& 3 U_{2 n}+2^{7} n(2 n-1)(-16(n-1)-42) \\
& \quad \equiv 2\left(7^{2 n}+6^{2 n}-4^{2 n}-3^{2 n}+1\right)-23 \cdot 2^{13} n(n-1) \quad\left(\bmod 2^{\alpha+14}\right)
\end{aligned}
$$

As $2 n \geq \alpha+13$,

$$
6^{2 n} \equiv 4^{2 n} \equiv 0 \quad\left(\bmod 2^{\alpha+13}\right)
$$


We also note that $2^{7} n(2 n-1)(16(n-1)+42)=2^{8}\left(16 n^{3}+18 n^{2}-13 n\right)$. Now, from the above we deduce that

$$
3 U_{2 n} \equiv 2^{8}\left(16 n^{3}+18 n^{2}-13 n\right)+2\left(7^{2 n}-3^{2 n}+1\right)-2^{13} n(n-1) \quad\left(\bmod 2^{\alpha+14}\right) .
$$

For $k \geq 5$ we see that $4 k-14>k \geq \operatorname{ord}_{2} k$. Thus, $2^{4 k} / k \equiv 0\left(\bmod 2^{14}\right)$ for $k \geq 4$. Hence

$$
\begin{aligned}
7^{2 n} & =(1+48)^{n}=1+\sum_{k=1}^{n} n\left(\begin{array}{l}
n-1 \\
k-1
\end{array}\right) \frac{48^{k}}{k} \\
& \equiv 1+48\left(\begin{array}{l}
n \\
1
\end{array}\right)+48^{2}\left(\begin{array}{l}
n \\
2
\end{array}\right)+48^{3}\left(\begin{array}{l}
n \\
3
\end{array}\right) \quad\left(\bmod 2^{\alpha+14}\right) .
\end{aligned}
$$

For $k \geq 7$ we have $3 k-14 \geq k \geq \operatorname{ord}_{2} k$. Thus $2^{3 k} / k \equiv 0\left(\bmod 2^{14}\right)$ for $k \geq 5$. Hence

$$
\begin{aligned}
3^{2 n} & =(1+8)^{n}=1+\sum_{k=1}^{n} n\left(\begin{array}{l}
n-1 \\
k-1
\end{array}\right) \frac{8^{k}}{k} \\
& \equiv 1+8\left(\begin{array}{l}
n \\
1
\end{array}\right)+8^{2}\left(\begin{array}{l}
n \\
2
\end{array}\right)+8^{3}\left(\begin{array}{l}
n \\
3
\end{array}\right)+8^{4}\left(\begin{array}{l}
n \\
4
\end{array}\right) \quad\left(\bmod 2^{\alpha+14}\right) .
\end{aligned}
$$

Therefore,

$$
\begin{aligned}
7^{2 n}-3^{2 n} & \equiv(48-8)\left(\begin{array}{l}
n \\
1
\end{array}\right)+\left(48^{2}-8^{2}\right)\left(\begin{array}{l}
n \\
2
\end{array}\right)+\left(48^{3}-8^{3}\right)\left(\begin{array}{l}
n \\
3
\end{array}\right)-8^{4}\left(\begin{array}{l}
n \\
4
\end{array}\right) \\
& \equiv 40 n+1120\left(n^{2}-n\right)-768 n(n-1)(n-2)-1536 n(n-1(n-2)(n-3) \\
& =-2^{9} \cdot 3 n^{4}+2^{8} \cdot 33 n^{3}-2^{5} \cdot 421 n^{2}+6600 n \\
& \equiv-2^{9} \cdot 3 n^{4}+2^{8} n^{3}+2^{5} \cdot 91 n^{2}-1592 n \quad\left(\bmod 2^{\alpha+13}\right) .
\end{aligned}
$$

This together with (3.4) yields

$$
\begin{aligned}
3 U_{2 n} \equiv & 2^{8}\left(16 n^{3}+18 n^{2}-13 n\right)+2\left(-2^{9} \cdot 3 n^{4}+2^{8} n^{3}\right. \\
& \left.\quad+2^{5} \cdot 91 n^{2}-1592 n+1\right)-2^{13} n(n-1) \\
= & -2^{10} \cdot 3 n^{4}+2^{9} \cdot 9 n^{3}+2^{6} \cdot 35 n^{2}+1680 n+2 \\
= & -3072 n^{4}+4608 n^{3}+2240 n^{2}+1680 n+2 \quad\left(\bmod 2^{\alpha+14}\right) .
\end{aligned}
$$

This proves the theorem.

Lemma 3.3. Let $k, m, b \in \mathbb{N}$ with $2 \mid b$. Then

$$
U_{2^{m} k+b}-U_{b} \equiv \frac{2^{b+1}}{9}-\frac{2}{3} \sum_{r=1}^{(b / 2)-1}\left(\begin{array}{c}
b \\
2 r
\end{array}\right) 2^{2 r}\left(U_{2^{m} k+b-2 r}-U_{b-2 r}\right) \quad\left(\bmod 2^{m+3}\right) .
$$

Proof. From [8, (4.1)],

$$
U_{2 n}=\frac{2}{3}\left(1-\sum_{r=1}^{n}\left(\begin{array}{l}
2 n \\
2 r
\end{array}\right) 2^{2 r} U_{2 n-2 r}\right)
$$


Thus,

$$
U_{b}=\frac{2}{3}\left(1-\sum_{r=1}^{b / 2}\left(\begin{array}{c}
b \\
2 r
\end{array}\right) 2^{2 r} U_{b-2 r}\right)
$$

and

$$
\begin{aligned}
U_{2^{m} k+b}=\frac{2}{3}( & 1-2^{2^{m} k+b} U_{0}-\sum_{r=1}^{2^{m-1} k+(b / 2)-1}\left(2^{m} k+b\right) \cdots \\
& \left.\times\left(2^{m} k+b-2 r+1\right) \cdot \frac{2^{2 r}}{(2 r) !} U_{2^{m} k+b-2 r}\right) .
\end{aligned}
$$

By (3.2), $2^{2 r} /(2 r) ! \equiv 0(\bmod 2)$ for $r \geq 1$. By (1.1), $2 \mid U_{2 n}$ for $n \geq 1$. We also have $2^{m} k+b \geq m+2$. Thus, from the above we deduce that

$$
\begin{aligned}
U_{2^{m} k+b} & \equiv \frac{2}{3}\left(1-\sum_{r=1}^{2^{m-1} k+(b / 2)-1} b(b-1) \cdots(b-2 r+1) \frac{2^{2 r}}{(2 r) !} U_{2^{m} k+b-2 r}\right) \\
& =\frac{2}{3}\left(1-\sum_{r=1}^{b / 2}\left(\begin{array}{c}
b \\
2 r
\end{array}\right) 2^{2 r} U_{2^{m} k+b-2 r}\right) \quad\left(\bmod 2^{m+3}\right) .
\end{aligned}
$$

Therefore,

$$
U_{2^{m} k+b}-U_{b} \equiv-\frac{2}{3} \sum_{r=1}^{b / 2}\left(\begin{array}{c}
b \\
2 r
\end{array}\right) 2^{2 r}\left(U_{2^{m} k+b-2 r}-U_{b-2 r}\right) \quad\left(\bmod 2^{m+3}\right) .
$$

By (3.1), $U_{2^{m} k} \equiv 48 \cdot 2^{m-1} k+\frac{2}{3} \equiv \frac{2}{3}\left(\bmod 2^{m+2}\right)$ for $2^{m} k \geq 6$. When $2^{m} k=2$ or 4 we also have $U_{2^{m} k} \equiv \frac{2}{3}\left(\bmod 2^{m+2}\right)$. Thus

$$
\begin{aligned}
U_{2^{m} k+b}-U_{b} & \equiv-\frac{2^{b+1}}{3}\left(U_{2^{m} k}-1\right)-\frac{2}{3} \sum_{r=1}^{(b / 2)-1}\left(\begin{array}{c}
b \\
2 r
\end{array}\right) 2^{2 r}\left(U_{2^{m} k+b-2 r}-U_{b-2 r}\right) \\
& \equiv \frac{2^{b+1}}{9}-\frac{2}{3} \sum_{r=1}^{(b / 2)-1}\left(\begin{array}{c}
b \\
2 r
\end{array}\right) 2^{2 r}\left(U_{2^{m} k+b-2 r}-U_{b-2 r}\right) \quad\left(\bmod 2^{m+3}\right),
\end{aligned}
$$

as required.

THEOREM 3.4. Let $k, m \in \mathbb{N}$.

(i) If $b \in\{2,4,6, \ldots\}$, then

$$
U_{2^{m} k+b} \equiv U_{b}+2^{b+1} \quad\left(\bmod 2^{\min \{b, m\}+3}\right) .
$$

(ii) We have

$$
U_{2^{m} k+2} \equiv-\frac{10}{9} \quad\left(\bmod 2^{m+3}\right) \quad \text { and } \quad U_{2^{m} k+4} \equiv \frac{34}{3} \quad\left(\bmod 2^{m+3}\right) .
$$


(iii) If $b \in\{4,6,8, \ldots\}$ and $b \leq m-2$, then

$$
U_{2^{m} k+b} \equiv U_{b}+2^{b+1}(4 b+5) \quad\left(\bmod 2^{b+5}\right) .
$$

Proof. If $b \in\{2,4,6, \ldots\}$, by Lemma 3.3 ,

$$
\begin{aligned}
U_{2^{m} k+b}-U_{b}-\frac{2^{b+1}}{9} \equiv- & \frac{2}{3} \sum_{r=1}^{(b / 2)-1}\left(\begin{array}{c}
b \\
2 r
\end{array}\right) 2^{2 r}\left(U_{2^{m} k+b-2 r}-U_{b-2 r}-\frac{2^{b-2 r+1}}{9}\right) \\
& -\frac{2}{3} \cdot \frac{2^{b+1}}{9} \sum_{r=1}^{(b / 2)-1}\left(\begin{array}{c}
b \\
2 r
\end{array}\right) \\
=- & \frac{2}{3} \sum_{r=1}^{(b / 2)-1}\left(\begin{array}{c}
b \\
2 r
\end{array}\right) 2^{2 r}\left(U_{2^{m} k+b-2 r}-U_{b-2 r}-\frac{2^{b-2 r+1}}{9}\right) \\
& -\frac{2^{b+2}}{27}\left(2^{b-1}-2\right)\left(\bmod 2^{m+3}\right) .
\end{aligned}
$$

Hence,

$$
\begin{aligned}
& U_{2^{m} k+b}-U_{b}-\frac{2^{b+1}}{9} \\
& \equiv-\frac{2}{3} \sum_{r=1}^{(b / 2)-1}\left(\begin{array}{c}
b \\
2 r
\end{array}\right) 2^{2 r}\left(U_{2^{m} k+b-2 r}-U_{b-2 r}-\frac{2^{b-2 r+1}}{9}\right) \quad\left(\bmod 2^{\min \{b, m\}+3}\right) .
\end{aligned}
$$

Therefore, for $b=2, U_{2^{m} k+b}-U_{b}-2^{b+1} / 9 \equiv 0\left(\bmod 2^{\min \{b, m\}+3}\right)$. We now prove (i) by induction on $b$. Suppose that the congruence

$$
U_{2^{m} k+b-2 r}-U_{b-2 r}-\frac{2^{b-2 r+1}}{9} \equiv 0 \quad\left(\bmod 2^{\min \{m, b-2 r\}+3}\right)
$$

holds for $r=1,2, \ldots,(b / 2)-1$. As

$$
2 \cdot 2^{2 r} \cdot 2^{\min \{m, b-2 r\}+3} \equiv \begin{cases}2 \cdot 2^{2 r} \cdot 2^{b-2 r+3} \equiv 0 \quad\left(\bmod 2^{b+3}\right) & \text { if } b \leq m, \\ 2 \cdot 2^{m+1} \cdot 2^{3} \equiv 0 \quad\left(\bmod 2^{m+3}\right) & \text { if } b>m \text { and } 2 r>m, \\ 2 \cdot 2^{2 r} \cdot 2^{m-2 r+3} \equiv 0 \quad\left(\bmod 2^{m+3}\right) & \text { if } b>m \geq 2 r\end{cases}
$$

from the above and induction we deduce $U_{2^{m} k+b}-U_{b}-2^{b+1} / 9 \equiv 0\left(\bmod 2^{\min \{b, m\}+3}\right)$. This yields (i).

We now consider (ii). Putting $b=2$ in Lemma 3.3, we see that

$$
U_{2^{m} k+2} \equiv U_{2}+\frac{2^{3}}{9}=-2+\frac{8}{9}=-\frac{10}{9} \quad\left(\bmod 2^{m+3}\right) .
$$

Taking $b=4$ in Lemma 3.3 and then applying the above, we deduce that

$$
U_{2^{m} k+4}-U_{4} \equiv \frac{2^{5}}{9}-\frac{2}{3}\left(\begin{array}{l}
4 \\
2
\end{array}\right) \cdot 2^{2}\left(U_{2^{m} k+2}-U_{2}\right) \equiv \frac{32}{9}-16 \cdot \frac{8}{9}=-\frac{32}{3} \quad\left(\bmod 2^{m+3}\right)
$$

and so $U_{2^{m} k+4} \equiv U_{4}-\frac{32}{3}=22-\frac{32}{3}=\frac{34}{3}\left(\bmod 2^{m+3}\right)$. This proves (ii). 
Finally, we consider (iii). Assume that $2 \leq b \leq m-2$. By (i), for $1 \leq r \leq(b / 2)-1$,

$$
U_{2^{m} k+b-2 r}-U_{b-2 r}-\frac{2^{b-2 r+1}}{9} \equiv 0 \quad\left(\bmod 2^{b-2 r+3}\right) .
$$

Thus, it follows from (3.5) that

$$
U_{2^{m} k+b}-U_{b}-\frac{2^{b+1}}{9} \equiv-\frac{2^{b+2}}{27}\left(2^{b-1}-2\right) \equiv 2^{2 b+1}-2^{b+3} \quad\left(\bmod 2^{b+4}\right) .
$$

Using this and (3.5),

$$
\begin{aligned}
& U_{2^{m} k+b}-U_{b}-\frac{2^{b+1}}{9} \equiv-\frac{2}{3} \sum_{r=1}^{(b / 2)-1}\left(\begin{array}{c}
b \\
2 r
\end{array}\right) 2^{2 r}\left(2^{2(b-2 r)+1}-2^{b-2 r+3}\right)-\frac{2^{b+3}}{27}\left(2^{b-2}-1\right) \\
& =-\frac{2^{b+2}}{3} \sum_{r=1}^{(b / 2)-1}\left(\begin{array}{c}
b \\
2 r
\end{array}\right) 2^{b-2 r}+\frac{2^{b+4}}{3} \sum_{r=1}^{(b / 2)-1}\left(\begin{array}{c}
b \\
2 r
\end{array}\right)-\frac{2^{2 b+1}}{27}+\frac{2^{b+3}}{27} \\
& \equiv\left\{\begin{array}{ccc}
-\frac{2^{b+2}}{3}\left(\begin{array}{c}
b \\
b-2
\end{array}\right) \cdot 2^{2}+\frac{2^{b+4}}{3}\left(2^{b-1}-2\right)-2^{b+3} & \\
\equiv 2^{b+3}(b-1) & \left(\bmod 2^{b+5}\right) & \text { if } b>2, \\
0 \quad\left(\bmod 2^{b+5}\right) & \text { if } b=2
\end{array}\right.
\end{aligned}
$$

and therefore for $b>2$,

$$
U_{2^{m} k+b}-U_{b} \equiv \frac{2^{b+1}}{9}+2^{b+3}(b-1) \equiv 2^{b+1}(4 b+5) \quad\left(\bmod 2^{b+5}\right) .
$$

This proves (iii). The proof is now complete.

Corollary 3.5. Let $k, m, b \in \mathbb{N}$ with $2 \mid b$. Then $U_{2^{m} k+b} \equiv U_{b}\left(\bmod 2^{\min \{b, m\}+1}\right)$.

Proof. This is immediate from Theorem 3.4(i).

\section{A congruence for $U_{k \varphi\left(3^{m}\right)+b}\left(\bmod 3^{m+4}\right)$}

In [6] the author proved that for $k, m \in \mathbb{N}, m \geq 4$ and $b \in\{0,2,4, \ldots\}$,

$$
E_{2^{m} k+b}-E_{b} \equiv\left\{\begin{array}{lll}
5 \cdot 2^{m} k & \left(\bmod 2^{m+4}\right) & \text { if } b \equiv 0,6 \quad(\bmod 8), \\
-3 \cdot 2^{m} k & \left(\bmod 2^{m+4}\right) & \text { if } b \equiv 2,4 \quad(\bmod 8) .
\end{array}\right.
$$

A generalisation to Euler polynomials was given in [7, Theorem 3.3]. 
From the proof of [8, Theorem 4.2] we have the following lemma.

LeMma 4.1. For $n \in \mathbb{N}$,

$$
2^{2 n} U_{2 n}=\sum_{k=0}^{n}\left(\begin{array}{l}
2 n \\
2 k
\end{array}\right) 3^{2 k} E_{2 k} \text {. }
$$

Theorem 4.2. Let $k, m \in \mathbb{N}, m \geq 3$ and $b \in\{0,2,4, \ldots\}$. Then

$$
U_{k \varphi\left(3^{m}\right)+b}-U_{b} \equiv \begin{cases}3^{m} k(9 b-40)\left(\bmod 3^{m+4}\right) & \text { if } 3 \mid b, \\ -3^{m} k \cdot 22\left(\bmod 3^{m+4}\right) & \text { if } 3 \mid b-1, \\ -3^{m} k(9 b-32)\left(\bmod 3^{m+4}\right) & \text { if } 3 \mid b-2 .\end{cases}
$$

Proof. By (3.2), $\operatorname{ord}_{3}(2 r) ! \leq r-1$. Thus, for $r \geq 3$,

$$
2 r-\operatorname{ord}_{3}(2 r) ! \geq 2 r-(r-1)=r+1 \geq 4
$$

and so $3^{2 r} /(2 r) ! \equiv 0\left(\bmod 3^{4}\right)$. Clearly $3^{2} \mid b(b-1) \cdots(b-2 r+1)$ for $r \geq 3$. Thus, for $r \geq 3$,

$$
\begin{aligned}
\left(k \varphi\left(3^{m}\right)+b\right)\left(k \varphi\left(3^{m}\right)+b-1\right) \cdots\left(k \varphi\left(3^{m}\right)+b-2 r+1\right) \\
\quad \equiv b(b-1) \cdots(b-2 r+1)+k \varphi\left(3^{m}\right) \sum_{i=0}^{2 r-1} \frac{b(b-1) \cdots(b-2 r+1)}{b-i} \\
\quad \equiv b(b-1) \cdots(b-2 r+1) \quad\left(\bmod 3^{m}\right) .
\end{aligned}
$$

Hence,

$$
\left(\begin{array}{c}
k \varphi\left(3^{m}\right)+b \\
2 r
\end{array}\right) 3^{2 r} \equiv\left(\begin{array}{c}
b \\
2 r
\end{array}\right) 3^{2 r} \quad\left(\bmod 3^{m+4}\right) \quad \text { for } r \geq 3 .
$$

Since $E_{0}=1, E_{2}=-1$ and $E_{4}=5$, using (4.1) and Lemma 4 .1 we see that

$$
\begin{aligned}
2^{k \varphi\left(3^{m}\right)+b} U_{k \varphi\left(3^{m}\right)+b}=1 & -9\left(\begin{array}{c}
k \varphi\left(3^{m}\right)+b \\
2
\end{array}\right)+5 \cdot 3^{4}\left(\begin{array}{c}
k \varphi\left(3^{m}\right)+b \\
4
\end{array}\right) \\
& +\sum_{r=3}^{\left(k \varphi\left(3^{m}\right)+b\right) / 2}\left(\begin{array}{c}
k \varphi\left(3^{m}\right)+b \\
2 r
\end{array}\right) 3^{2 r} E_{2 r} \\
\equiv- & \frac{9}{2}\left(4 \cdot 3^{2 m-2} k^{2}+2 \cdot 3^{m-1} k(2 b-1)\right)+\frac{3^{3} \cdot 5}{8}\left(k^{4} \varphi\left(3^{m}\right)^{4}\right. \\
& +(4 b-6) k^{3} \varphi\left(3^{m}\right)^{3}+\left(6 b^{2}-18 b+11\right) k^{2} \varphi\left(3^{m}\right)^{2} \\
& \left.+\left(4 b^{3}-18 b^{2}+22 b-6\right) k \varphi\left(3^{m}\right)\right)+\sum_{r=0}^{b / 2}\left(\begin{array}{c}
b \\
2 r
\end{array}\right) 3^{2 r} E_{2 r} \\
\equiv-2 & \cdot 3^{2 m} k^{2}-(2 b-1) 3^{m+1} k-\left(4 b^{3}+4 b-6\right) 3^{m+2} k \\
& +2^{b} U_{b}\left(\bmod 3^{m+4}\right) .
\end{aligned}
$$


By (3.2), $\operatorname{ord}_{3} r \leq \operatorname{ord}_{3} r$ ! $<r / 2$. Thus, for $r \geq 4$,

$$
2 r-\operatorname{ord}_{3} r>2 r-\frac{r}{2}=\frac{3 r}{2} \geq 6
$$

and so

$$
\varphi\left(3^{m-1}\right) \frac{9^{r}}{r}=2 \cdot 3^{m-2} \cdot \frac{3^{2 r}}{r} \equiv 0 \quad\left(\bmod 3^{m+4}\right)
$$

Hence,

$$
\begin{aligned}
& 2^{k \varphi\left(3^{m}\right)}-1=(1-9)^{k \varphi\left(3^{m-1}\right)}-1=\sum_{r=1}^{k \varphi\left(3^{m-1}\right)}\left(\begin{array}{c}
k \varphi\left(3^{m-1}\right) \\
r
\end{array}\right)(-9)^{r} \\
&=\left.\begin{array}{c}
k \varphi\left(3^{m-1}\right) \\
1
\end{array}\right)(-9)+\left(\begin{array}{c}
k \varphi\left(3^{m-1}\right) \\
2
\end{array}\right)(-9)^{2}+\left(\begin{array}{c}
k \varphi\left(3^{m-1}\right) \\
3
\end{array}\right)(-9)^{3} \\
&+\sum_{r=4}^{k \varphi\left(3^{m-1}\right)} k \varphi\left(3^{m-1}\right)\left(\begin{array}{c}
k \varphi\left(3^{m-1}\right)-1 \\
r-1
\end{array}\right) \frac{(-9)^{r}}{r} \\
& \equiv-9 k \varphi\left(3^{m-1}\right)+81 \frac{k \varphi\left(3^{m-1}\right)\left(k \varphi\left(3^{m-1}\right)-1\right)}{2} \quad+\frac{k \varphi\left(3^{m-1}\right)\left(k \varphi\left(3^{m-1}\right)-1\right)\left(k \varphi\left(3^{m-1}\right)-2\right)}{6}(-9)^{3} \\
& \equiv 3^{m} k\left(16+2 \cdot 3^{m} k\right) \quad\left(\bmod 3^{m+4}\right)
\end{aligned}
$$

Thus,

$$
\begin{aligned}
2^{k \varphi\left(3^{m}\right)+b} U_{k \varphi\left(3^{m}\right)+b}-2^{b} U_{b} \\
\quad \equiv\left(1+3^{m} k\left(16+2 \cdot 3^{m} k\right)\right) 2^{b} U_{k \varphi\left(3^{m}\right)+b}-2^{b} U_{b} \\
\quad=2^{b}\left(U_{k \varphi\left(3^{m}\right)+b}-U_{b}\right)+3^{m} k\left(16+2 \cdot 3^{m} k\right) 2^{b} U_{k \varphi\left(3^{m}\right)+b} \quad\left(\bmod 3^{m+4}\right) .
\end{aligned}
$$

By Lemma 4.1,

$$
2^{2 n} U_{2 n} \equiv E_{0}+\left(\begin{array}{c}
2 n \\
2
\end{array}\right) 3^{2} E_{2}=1-9 n(2 n-1) \quad(\bmod 81) .
$$

Thus,

$$
2^{k \varphi\left(3^{m}\right)+b} U_{k \varphi\left(3^{m}\right)+b} \equiv 1-\frac{9}{2}\left(k \varphi\left(3^{m}\right)+b\right)\left(k \varphi\left(3^{m}\right)+b-1\right) \equiv 1-9\left(\begin{array}{l}
b \\
2
\end{array}\right) \quad(\bmod 81)
$$

and so

$$
\begin{aligned}
2^{b} U_{k \varphi\left(3^{m}\right)+b} & \equiv \frac{1-9\left(\begin{array}{c}
b \\
2
\end{array}\right)}{2^{k \varphi\left(3^{m}\right)}} \\
& \equiv \frac{1-\frac{9}{2} b(b-1)}{1+3^{m} k\left(16+2 \cdot 3^{m} k\right)}
\end{aligned}
$$




$$
\begin{aligned}
& \equiv\left(1-\frac{9}{2} b(b-1)\right)\left(1-3^{m} k\left(16+2 \cdot 3^{m} k\right)\right) \\
& \equiv 1-\frac{9}{2} b(b-1)-16 \cdot 3^{m} k \quad(\bmod 81) .
\end{aligned}
$$

Therefore,

$$
\begin{aligned}
& -2 \cdot 3^{2 m} k^{2}-(2 b-1) 3^{m+1} k-\left(4 b^{3}+4 b-6\right) 3^{m+2} k \\
& \equiv 2^{k \varphi\left(3^{m}\right)+b} U_{k \varphi\left(3^{m}\right)+b}-2^{b} U_{b} \equiv 2^{b}\left(U_{k \varphi\left(3^{m}\right)+b}-U_{b}\right)+3^{m} k\left(16+2 \cdot 3^{m} k\right) 2^{b} U_{k \varphi\left(3^{m}\right)+b} \\
& \equiv 2^{b}\left(U_{k \varphi\left(3^{m}\right)+b}-U_{b}\right)+3^{m} k\left(16+2 \cdot 3^{m} k\right)\left(1-\frac{9}{2} b(b-1)-16 \cdot 3^{m} k\right) \\
& \equiv \begin{cases}2^{b}\left(U_{k \varphi\left(3^{m}\right)+b}-U_{b}\right)+3^{m} k(16+9 b(b-1))\left(\bmod 3^{m+4}\right) & \text { if } m \geq 4, \\
2^{b}\left(U_{k \varphi\left(3^{3}\right)+b}-U_{b}\right)+27 k(16+9 b(b-1))+3^{6} k^{2} \quad\left(\bmod 3^{7}\right) & \text { if } m=3 .\end{cases}
\end{aligned}
$$

This yields

$$
2^{b}\left(U_{k \varphi\left(3^{m}\right)+b}-U_{b}\right) \equiv-3^{m} k\left(36 b^{3}+9 b^{2}+33 b-41\right) \quad\left(\bmod 3^{m+4}\right) .
$$

If $3 \mid b$, then $2^{-b}=(1-9)^{-b / 3}$ and so

$$
\begin{aligned}
U_{k \varphi\left(3^{m}\right)+b}-U_{b} & \equiv(1-9)^{-b / 3}\left(-3^{m} k\right)\left(36 b^{3}+9 b^{2}+33 b-41\right) \\
& \equiv-(1+3 b)(33 b-41) 3^{m} k \equiv(9 b-40) 3^{m} k \quad\left(\bmod 3^{m+4}\right) .
\end{aligned}
$$

If $3 \mid b-1, \quad$ then $b^{2} \equiv 2 b-1(\bmod 9), \quad b^{3} \equiv 1(\bmod 9) \quad$ and $\quad 2^{-b}=4 \cdot 2^{-b-2}=$ $4(1-9)^{-(b+2) / 3}$. Thus,

$$
\begin{aligned}
U_{k \varphi\left(3^{m}\right)+b}-U_{b} & \equiv 4(1-9)^{-(b+2) / 3}\left(-3^{m} k\right)\left(36 b^{3}+9 b^{2}+33 b-41\right) \\
& \equiv-4(1+3(b+2))(36+9(2 b-1)+33 b-41) 3^{m} k \\
& =-4(3 b+7)(51 b-14) 3^{m} k \equiv 8(3 b+7)(15 b+7) 3^{m} k \\
& \equiv 8(45(2 b-1)+126 b+49) 3^{m} k=8(216 b+4) 3^{m} k \\
& \equiv 8(216+4) 3^{m} k \equiv-22 \cdot 3^{m} k \quad\left(\bmod 3^{m+4}\right) .
\end{aligned}
$$

If $3 \mid b-2$, then $b^{2} \equiv-2 b-1(\bmod 9), \quad b^{3} \equiv-1(\bmod 9)$ and $2^{-b}=2 \cdot 2^{-b-1}=$ $-2(1-9)^{-(b+1) / 3}$. Thus,

$$
\begin{aligned}
U_{k \varphi\left(3^{m}\right)+b}-U_{b} & \equiv-2(1-9)^{-(b+1) / 3}\left(-3^{m} k\right)\left(36 b^{3}+9 b^{2}+33 b-41\right) \\
& \equiv 2(1+3(b+1))(-36+9(-2 b-1)+33 b-41) 3^{m} k \\
& \equiv 10(3 b+4)(3 b-1) 3^{m} k \equiv 10(9(-2 b-1)+9 b-4) 3^{m} k \\
& \equiv 10(-9 b-13) 3^{m} k \equiv-(9 b-32) 3^{m} k \quad\left(\bmod 3^{m+4}\right) .
\end{aligned}
$$

This completes the proof. 


\section{A congruence for $E_{k \varphi\left(3^{m}\right)+b}\left(\bmod 3^{m+4}\right)$}

Lemma 5.1. For $n \in \mathbb{N}$,

$$
\left(3^{2 n}+1\right) E_{2 n}=\sum_{r=0}^{n}\left(\begin{array}{l}
2 n \\
2 r
\end{array}\right) 2^{2 n-2 r+1} 3^{2 r} E_{2 r} .
$$

Proof. By [7, Theorem 2.1 and Lemma 2.1],

$$
\frac{1}{2}\left(3^{2 n}+1\right) E_{2 n}=\sum_{r=0}^{n}\left(\begin{array}{l}
2 n \\
2 r
\end{array}\right)(1-3)^{2 n-2 r} 3^{2 r} E_{2 r}
$$

as required.

Let $k, m \in \mathbb{N}$ and $b \in\{0,2,4, \ldots\}$. From [2, p. 231] or [5, Corollary 7.1],

$$
E_{k \varphi\left(3^{m}\right)+b} \equiv\left(3^{b}+1\right) E_{b} \quad\left(\bmod 3^{m}\right) .
$$

Now we prove the following stronger congruence.

Theorem 5.2. Let $k, m \in \mathbb{N}, m \geq 3$ and $b \in\{0,2,4, \ldots\}$. Then

$$
E_{k \varphi\left(3^{m}\right)+b}-\left(3^{b}+1\right) E_{b} \equiv \begin{cases}(9 b+20) 3^{m} k\left(\bmod 3^{m+4}\right) & \text { if } 3 \mid b, \\ -16 \cdot 3^{m} k\left(\bmod 3^{m+4}\right) & \text { if } 3 \mid b-1, \\ (-9 b+11) 3^{m} k\left(\bmod 3^{m+4}\right) & \text { if } 3 \mid b-2 .\end{cases}
$$

Proof. As $\varphi\left(3^{m}\right) \geq m+4$, using Lemma 5.1 and (4.1) we see that

$$
\begin{aligned}
E_{k \varphi\left(3^{m}\right)+b} \equiv & \left(3^{k \varphi\left(3^{m}\right)+b}+1\right) E_{k \varphi\left(3^{m}\right)+b} \\
= & \sum_{r=0}^{\left(k \varphi\left(3^{m}\right)+b\right) / 2}\left(\begin{array}{c}
k \varphi\left(3^{m}\right)+b \\
2 r
\end{array}\right) 2^{k \varphi\left(3^{m}\right)+b-2 r+1} 3^{2 r} E_{2 r} \\
= & 2^{k \varphi\left(3^{m}\right)+b+1} E_{0}+\left(\begin{array}{c}
k \varphi\left(3^{m}\right)+b \\
2
\end{array}\right) 2^{k \varphi\left(3^{m}\right)+b-1} 3^{2} E_{2} \\
& +\left(\begin{array}{c}
k \varphi\left(3^{m}\right)+b \\
4
\end{array}\right) 2^{k \varphi\left(3^{m}\right)+b-3} 3^{4} E_{4} \\
& +\sum_{r=3}^{b / 2}\left(\begin{array}{c}
b \\
2 r
\end{array}\right) 2^{k \varphi\left(3^{m}\right)+b-2 r+1} 3^{2 r} E_{2 r} \quad\left(\bmod 3^{m+4}\right) .
\end{aligned}
$$

From the proof of Theorem 4.2 we know that $3^{2 r} /(2 r) ! \equiv 0\left(\bmod 3^{4}\right)$ for $r \geq 3$. By Euler's theorem, $2^{k \varphi\left(3^{m}\right)} \equiv 1\left(\bmod 3^{m}\right)$. Thus, from the above we deduce that

$$
\begin{array}{r}
E_{k \varphi\left(3^{m}\right)+b} \equiv 2^{k \varphi\left(3^{m}\right)+b+1}-9\left(k \varphi\left(3^{m}\right)+b\right)\left(k \varphi\left(3^{m}\right)+b-1\right) 2^{k \varphi\left(3^{m}\right)+b-2} \\
+\left(\begin{array}{c}
k \varphi\left(3^{m}\right)+b \\
4
\end{array}\right) 2^{b-3} \cdot 81 \cdot 5+\sum_{r=3}^{b / 2}\left(\begin{array}{c}
b \\
2 r
\end{array}\right) 2^{b-2 r+1} 3^{2 r} E_{2 r}
\end{array}
$$




$$
\begin{aligned}
& \equiv 2^{b+1}\left(2^{k \varphi\left(3^{m}\right)}-1\right)-9\left(k^{2} \varphi\left(3^{m}\right)^{2}+(2 b-1) k \varphi\left(3^{m}\right)+b(b-1)\right) 2^{k \varphi\left(3^{m}\right)+b-2} \\
& \quad+9 b(b-1) 2^{b-2}+\left(\left(\begin{array}{c}
k \varphi\left(3^{m}\right)+b \\
4
\end{array}\right)-\left(\begin{array}{c}
b \\
4
\end{array}\right)\right) 2^{b-3} \cdot 81 \cdot 5 \\
& \quad+\sum_{r=0}^{b / 2}\left(\begin{array}{c}
b \\
2 r
\end{array}\right) 2^{b-2 r+1} 3^{2 r} E_{2 r} \\
& \equiv 2^{b+1}\left(2^{k \varphi\left(3^{m}\right)}-1\right)-2^{b} k^{2} \cdot 3^{2 m}-9(2 b-1) 2^{b-2} k \varphi\left(3^{m}\right) \\
& \quad-9 b(b-1) 2^{b-2}\left(2^{k \varphi\left(3^{m}\right)}-1\right)+5 \cdot 3^{3} \cdot 2^{b-6}\left(4 b^{3}-18 b^{2}\right. \\
& \quad+22 b-6) k \varphi\left(3^{m}\right)+\left(3^{b}+1\right) E_{b} \\
& \equiv 2^{b-2}(8-9 b(b-1))\left(2^{k \varphi\left(3^{m}\right)}-1\right)-2^{b} k^{2} \cdot 3^{2 m}-(2 b-1) 2^{b-1} k \cdot 3^{m+1} \\
& \quad-2^{b-2}\left(2 b^{3}+2 b-3\right) k \cdot 3^{m+2}+\left(3^{b}+1\right) E_{b} \quad\left(\bmod 3^{m+4}\right) .
\end{aligned}
$$

By the proof of Theorem 4.2,

$$
2^{k \varphi\left(3^{m}\right)}-1 \equiv 16 k \cdot 3^{m}+2 k^{2} \cdot 3^{2 m}\left(\bmod 3^{m+4}\right) .
$$

Thus,

$$
\begin{aligned}
& E_{k \varphi\left(3^{m}\right)+b}-\left(3^{b}+1\right) E_{b} \equiv 2^{b-2} 3^{m} k\left\{16\left(8-9 b^{2}+9 b\right)-6(2 b-1)-9\left(2 b^{3}+2 b-3\right)\right\}+2^{b-2}(8-9 b(b-1)) \cdot 2 k^{2} 3^{2 m}-2^{b} k^{2} 3^{2 m} \\
& \equiv 2^{b-2}\left(-18 b^{3}+18 b^{2}-48 b-1\right) 3^{m} k \quad\left(\bmod 3^{m+4}\right) .
\end{aligned}
$$

If $3 \mid b$, then $2^{b-2} \equiv-20(1-9)^{b / 3} \equiv-20(1-3 b)\left(\bmod 3^{4}\right)$. Thus,

$$
\begin{aligned}
E_{k \varphi\left(3^{m}\right)+b}-\left(3^{b}+1\right) E_{b} & \equiv-20(1-3 b)\left(-18 b^{3}+18 b^{2}-48 b-1\right) 3^{m} k \\
& \equiv 20(1-3 b)(1+48 b) 3^{m} k \\
& \equiv 20(1+45 b) 3^{m} k \equiv(9 b+20) 3^{m} k \quad\left(\bmod 3^{m+4}\right) .
\end{aligned}
$$

If $3 \mid b-1$, then $b^{2} \equiv 2 b-1(\bmod 9), b^{3} \equiv 1(\bmod 9)$ and

$$
2^{b-2}=-\frac{1}{2}(1-9)^{(b-1) / 3} \equiv-\frac{1}{2}\left(1-9 \cdot \frac{b-1}{3}\right)=\frac{1}{2}(3 b-4) \quad\left(\bmod 3^{4}\right) .
$$

Thus,

$$
\begin{aligned}
E_{k \varphi\left(3^{m}\right)+b}-\left(3^{b}+1\right) E_{b} & \equiv \frac{1}{2}(3 b-4)\left(-18 b^{3}+18 b^{2}-48 b-1\right) 3^{m} k \\
& \equiv \frac{1}{2}(3 b-4)(-18+18(2 b-1)-48 b-1) 3^{m} k \\
& \equiv(3 b-4)(-6 b+22) 3^{m} k \equiv\left(-18 b^{2}+9 b-7\right) 3^{m} k \\
& \equiv(-18(2 b-1)+9 b-7) 3^{m} k \\
& =(-27 b+11) 3^{m} k \equiv-16 \cdot 3^{m} k \quad\left(\bmod 3^{m+4}\right) .
\end{aligned}
$$


If $3 \mid b-2$, then $b^{2} \equiv-2 b-1(\bmod 9), b^{3} \equiv-1(\bmod 9)$ and $2^{b-2}=(1-9)^{(b-2) / 3} \equiv$ $1-9 \cdot(b-2) / 3=7-3 b\left(\bmod 3^{4}\right)$. Thus,

$$
\begin{aligned}
E_{k \varphi\left(3^{m}\right)+b}-\left(3^{b}+1\right) E_{b} & \equiv(7-3 b)\left(-18 b^{3}+18 b^{2}-48 b-1\right) 3^{m} k \\
& \equiv(7-3 b)(18+18(-2 b-1)-48 b-1) 3^{m} k \\
& \equiv(3 b-7)(3 b+1) 3^{m} k \equiv(9(-2 b-1)-18 b-7) 3^{m} k \\
& \equiv(-9 b+11) 3^{m} k \quad\left(\bmod 3^{m+4}\right) .
\end{aligned}
$$

This completes the proof.

\section{References}

[1] H. Bateman, Higher Transcendental Functions, Vol. I (McGraw-Hill, New York, 1953).

[2] K. W. Chen, 'Congruences for Euler numbers', Fibonacci Quart. 42 (2004), 128-140.

[3] J. B. Cosgrave and K. Dilcher, 'Mod $p^{3}$ analogues of theorems of Gauss and Jacobi on binomial coefficients', Acta Arith. 142 (2010), 103-118.

[4] S. Mattarei and R. Tauraso, 'Congruences for central binomial sums and finite polylogarithms', J. Number Theory, to appear.

[5] Z. H. Sun, 'Congruences involving Bernoulli polynomials', Discrete Math. 308 (2008), 71-112.

[6] Z. H. Sun, 'Euler numbers modulo 2n', Bull. Aust. Math. Soc. 82 (2010), 221-231.

[7] Z. H. Sun, 'Congruences for sequences similar to Euler numbers', J. Number Theory 132 (2012), 675-700.

[8] Z. H. Sun, 'Identities and congruences for a new sequence', Int. J. Number Theory 8 (2012), 207-225.

ZHI-HONG SUN, School of Mathematical Sciences, Huaiyin Normal University, Huaian, Jiangsu 223001, PR China e-mail: zhihongsun@yahoo.com 